\title{
Highly Electrophilic Titania Hole as a Versatile and Efficient Pho- tochemical Free Radical Source
}

\author{
Andrew Hainer ${ }^{\dagger}$, Nancy Marina ${ }^{\dagger}$, Stefanie Rincon, Paolo Costa, Anabel E. Lanterna* and Juan C. Scaiano*
}

Department of Chemistry and Biomolecular Sciences and Centre for Advanced Materials Research (CAMaR), University of Ottawa, Ottawa, Canada K1N 6N5

\section{Supporting Information Placeholder}

\begin{abstract}
Photogenerated holes in nanometric semiconductors, such as $\mathrm{TiO}_{2}$, constitute remarkable powerful electrophilic centers, capable of capturing an electron from numerous donors such as ethers, or non-activated substrates 5 like toluene or acetonitrile, and constitute an exceptionally clean and efficient source of free radicals. In contrast with typical free radical precursors, semiconductors generate single radicals (rather than pairs), where the precursors can be readily removed by filtration or centrifugation after use, thus 10 making it a convenient tool in organic chemistry. The process can be described as an example of dystonic proton coupled electron transfer (PCET).
\end{abstract}

$\mathrm{C}-\mathrm{H}$ activation has gained much interest due to its atom and step-economy in the synthesis of functional molecules, ${ }^{1}$ many of them are of importance in drug development. Among these, the $\mathrm{sp}^{3} \boldsymbol{\alpha}-\mathrm{C}-\mathrm{H}$ reactions of heteroatomic compounds (e.g., alcohols, ethers, or amines) have particular synthetic value as they can directly introduce active groups. ${ }^{2}$ For example, the THF motif ranks 11 among the 100 most frequently used ring systems from small molecule drugs listed by FDA, ${ }^{3}$ and is part of eribulin and afatinib, two commercial cancer treatment drugs. ${ }^{4}$ Thus, the study of different methodologies to couple this and other structures to organic molecules has gained much attention. ${ }^{5}$ Two mechanisms proposed for radical C-H activation, involve the cleavage of a C$\mathrm{H}$ bond by hydrogen atom transfer (HAT) or a single-electron transfer, usually coupled to proton loss, described as proton coupled electron transfer (PCET), ${ }^{6}$ Scheme 1.



\section{Scheme 1. C-H activation via HAT and PCET.}

These mechanisms normally require the presence of a free radical initiator that generates two radicals, leaving initiator- derived debris in the solution along with residual initiator, which can be difficult to remove. Here, we demonstrate that 35 photoactivated $\mathrm{TiO}_{2}$ can be used as a clean free radical initiator, circumventing some of the disadvantages mentioned.

Many of the studies involving $\mathrm{TiO}_{2}$ radical formation deal with the generation of reactive oxygen species (ROS), whose involvement ranges from applications in environmental re40 mediation $^{7}$ to potential concerns when ROS are used in sunscreens. ${ }^{8}$ In contrast, our report is focused on the clean formation of free carbon-centered radicals for applications in organic chemistry. Two recent examples show that semiconductor-based photocatalysts can activate THF, ultimately 45 leading to $\mathrm{C}-\mathrm{C}$ coupling reactions. ${ }^{9}$ Here, we explore in detail the initiation reaction with various substrates. While ethers are not very good electron donors (for example, they show a broad electrochemical window $)^{10}$, it is remarkable that the photogenerated $\mathrm{TiO}_{2}$-hole is so electrophilic that it readily 50 oxidizes ethers. Indeed, it can also oxidize non-activated substrates such as toluene and acetonitrile. While most initiators generate a pair of free radicals, $\mathrm{TiO}_{2}$ makes a single radical therefore, cage recombination is not an issue. Further, residual $\mathrm{TiO}_{2}$ and any $\mathrm{H}_{2}$ produced are readily removed. While the

55 key reaction occurs at the hole (Scheme 2), the electron-hole recombination can be slowed down by decorating $\mathrm{TiO}_{2}$ with metal nanoparticles, one of the strategies explored here.

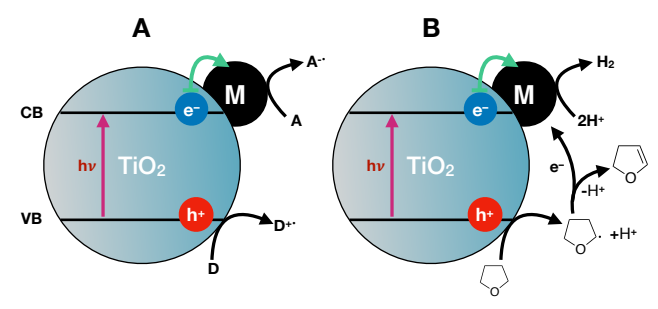

Scheme 2. A) Metal nanoparticles improve charge sepa60 ration in $\mathrm{TiO}_{2}$ semiconductors facilitating both, reduction $\left(A \rightarrow A^{-\cdot}\right)$ and oxidation $\left(D \rightarrow D^{+\cdot}\right)$ pathways. B) Proposed mechanism for ethers (illustrated for THF). 
Our experiments use $368 \mathrm{~nm}$ LED irradiation of metal decorated $\mathrm{TiO}_{2}$, such as $\mathrm{Pd} @ \mathrm{TiO}_{2}$, suspended in the reaction mixture. While our work concentrates on organic solvents, the reaction is also water-tolerant (Fig. S2). The photolysis generates $\mathrm{H}_{2}$ gas as a by-product, just as many sacrificial electron donors do. ${ }^{11}$ This gives the system the ability to remove $\mathrm{H}^{+}$from solution, making base unnecessary for reactions that 7o can withstand mild acidic conditions. Scheme 3 shows our proposed mechanism, where we anticipate that the hole will reside in the $\mathrm{TiO}_{2}$ valence band (VB), while the electrons will migrate rapidly to the metal particle, effectively their host. Dihydrofuran and furan were detected by NMR (Fig S11-S12).



Scheme 3: Mechanism for the formation of $\mathrm{H}_{2}$ and dihydrofuran from THF. THF-derived radicals (eq. 2) are mobile and undergo solution reactions.

The generation of radicals does not guarantee they are

"free", in the sense that they are mobile and can participate in solution reactions, as in some cases surface-generated intermediates can remain and react on the surface. In order to validate the mechanism and evaluate if the THF radical is truly free, we used TEMPO (2) as a free radical scavenger. Reaction 6 illustrates the process, where the product can be readily detected by gas chromatography.

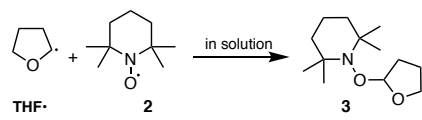

(6)

Product 3 is formed quantitatively in the presence of TEMPO and different $\mathrm{M} @ \mathrm{TiO}_{2}$ nanocomposites (Fig. 1). In eight different catalysts, including bare $\mathrm{TiO}_{2}$. Most of these materials were used in earlier reports and their properties are summarized in Table $\mathrm{S} 1$. We monitored the time required for $50 \%$ of the TEMPO to yield radical trapping products upon sults, where all decorated materials show similar performance, and are about five times more efficient than bare $\mathrm{TiO}_{2}$. This virtual independence of the electron trapping material supports the $\mathrm{TiO}_{2}$ hole as responsible for the electrophilic properties observed. It is clear that the dominant feature of these materials is that surface metals (or their oxides) can greatly increase the longevity of hole-electron pair, slowing down charge recombination and favoring trapping by molecules in the medium, such as THF in these examples.
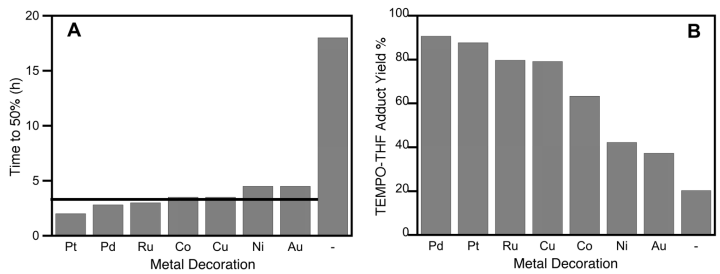

Figure 1. A: Time for $50 \%$ TEMPO to product 3 conversion under $368 \mathrm{~nm}$ irradiation. Horizontal line at $3.4 \mathrm{~h}$ represents average time for all $\mathrm{M} @ \mathrm{TiO}_{2}$ samples. Full kinetic details in Fig. S1. B: Yield of product 3 using various

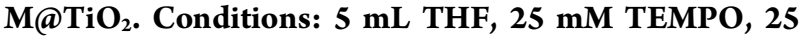
$\mathrm{mM} \mathrm{Cs}_{2} \mathrm{CO}_{3}, 20 \mathrm{mg}$ catalyst, Ar, hv at $2500 \mathrm{Wm}^{-2}, 4 \mathrm{~h}$.

Figure 2. Rates of photocatalytic $\mathrm{H}_{2}$ generation for $\mathrm{M} @ \mathrm{TiO}_{2}$ in the absence (black) and in the presence of TEMPO (grey). Reaction conditions as in Fig. 1.

For catalysts such as $\mathrm{Ru}, \mathrm{Ni}, \mathrm{Cu}$, or Co-decorated $\mathrm{TiO}_{2}$, the decorating structures are redox-active oxides that can find alternate pathways for $\mathrm{CB}$ electrons, ${ }^{12}$ not surprisingly affecting the reducing ability of the material (i.e., $\mathrm{H}_{2}$ formation). Similarly for cobalt, minor redox changes can affect extensively the outcome of the catalytic process. ${ }^{13}$

125 Having established that the THF reactivity of various $\mathrm{M} @ \mathrm{TiO}_{2}$ is similar among the materials tested, we explored a series of ethers to evaluate the generality of this approach. Note that ethers are known to react rapidly with alkoxyl radicals, another highly electrophilic species. ${ }^{14}$ The reaction responds to stereoelectronic effects and THF is one of the fastest reactants towards tert-butoxyl radicals. Our selection of ethers parallels the most interesting examples found for tertbutoxyl. ${ }^{14}$ We have also observed that $\mathrm{H}_{2}$ is an excellent way to screen for hole-electron reactivity, even if $\mathrm{H}_{2}$ is derived 135 from $\mathrm{CB}$ reactions (eq. 3 and 4). ${ }^{15}$ Table 1 summarizes the TEMPO-ether adduct formation for a series of ethers tested, and the $\mathrm{H}_{2}$ evolution in the absence of TEMPO. Given that many organic reactions are performed in the presence of base, we tested the $\mathrm{TiO}_{2}$ strategy under these conditions and 
conclude that the system is base-tolerant; indeed, TEMPOether adduct yields are slightly higher in basic media (Table S2), likely due to the decrease of TEMPO-side reactions. ${ }^{16}$

Table 1. Yields of TEMPO-ether adducts using $\mathrm{Pd} @ \mathrm{TiO}_{2}$ photocatalyst and $\mathrm{H}_{2}$ evolution in the absence of TEMPO

\begin{tabular}{|c|c|c|c|}
\hline $\begin{array}{l}\text { Ether } \\
\text { (label) }\end{array}$ & Structure & $\begin{array}{l}\text { TEMPO-Ether } \\
\text { Adduct Yield \% } \\
\end{array}$ & $\begin{array}{l}\mathbf{H}_{2} \\
\left(\mathbf{m m o l ~ g}^{-1} \mathbf{h}^{-1}\right)\end{array}$ \\
\hline THF & & 63.5 & 0.74 \\
\hline DEE & & 62.8 & 0.51 \\
\hline DOX & & 39.2 & 0.52 \\
\hline THP & & 28.2 & 0.72 \\
\hline DMM & & ND & 1.32 \\
\hline MOP & & ND & 1.14 \\
\hline DOL & & ND & 1.03 \\
\hline MDOL & & ND & 0.70 \\
\hline No ether & & -- & 0.0 \\
\hline
\end{tabular}

145 Ethers: THF (tetrahydrofuran), DEE (diethyl ether), DOX (dioxane), THP (tetrahydropyran), MOP (1-methoxypropane), DOL (1,3dioxolane), MDOL (2-methyl-1,3-dioxolane), and DMM (dimethoxymethane). ${ }^{a}$ Reaction conditions summarized in SI. Full conversion of TEMPO was obtained in all cases.

While TEMPO trapping works very well with THF and other molecules (e.g., dioxane), the product mixture can be complicated by side reactions in other systems, including some cases where the amine 2,2,6,6-tetramethylpiperidine is produced; TEMPO deoxygenation was reported ${ }^{16}$ and has been identified in other systems (see SI).

In many cases, $\mathrm{H}_{2}$ generation can be used to screen which ethers are good candidates as hole scavengers (Table 1). Interestingly, among the ethers studied the highest production of $\mathrm{H}_{2}$ was detected with some that did not show the forDOL, and MDOL). This behavior can be rationalized by understanding the chemistry of the radicals. After radical formation these ethers can break the $\mathrm{C}-\mathrm{O}$ bond to generate the corresponding aldehyde and the carbon-centered radical, for the generation of $\mathrm{H}_{2}$. In order to test this hypothesis, we monitored the formation of $\mathrm{CH}_{2} \mathrm{O}$ for the systems where this product is anticipated (i.e., DMM and MOP) and using THF as a (negative) control. Reaction 7 illustrates the cleavage for the radicals derived from DMM, and the corresponding enthalpy of reaction estimated from known thermodynamic data (Scheme S1). ${ }^{17}$ In fact, $\mathrm{CH}_{2} \mathrm{O}$ can be detected during the early stages of reaction, and quickly reaches a plateau at 8-10 mM, attributed to the fact that $\mathrm{CH}_{2} \mathrm{O}$ is an excellent SED, quickly reaching steady state concentration when it is consumed as fast as it is generated. Initial formaldehyde formation can be observed with an increasing rate of $\mathrm{H}_{2}$ generation within the first $15 \mathrm{~min}$ of the reaction (Fig. S4). Thus, $\mathrm{H}_{2}$ formation is a good reporter for hole trapping even when 180 TEMPO trapping fails due to product mix complexity.

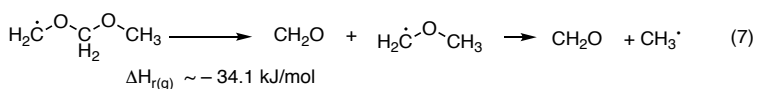

Further confirmation that the radicals are free and mobile was obtained by EPR spectroscopy (Fig. S5-S7) and in experiments with THF and dioxane, separate or in equimolar mixtures, where radical recombination products are easily observed, including the cross dimer in the case of solvent mixtures (Fig. S8-S10). In order to compare ether reactivities we also performed a few competitive studies between THF and two other ethers, that presented uncomplicated chemistry in 190 TEMPO trapping studies (Table S3). Whereas the $\mathrm{Bu}^{t} \mathrm{O}$ chemistry relies in stereoelectronic control, this is lost in the $\mathrm{TiO}_{2}$-hole chemistry. In the context of Table $\mathrm{S} 3, \mathrm{Bu}^{\mathrm{t}} \mathrm{O}^{*}$ is closer to a HAT mechanism, while PCET probably dominates the $\mathrm{TiO}_{2}$ chemistry. These results combine ether-hole 195 reactivities with surface preference affinities. Computational studies suggest that while dioxane has a modest reactivity, it does have the highest surface affinity. In mix reagents this can lower the relative production of THF radicals in the presence of dioxane due to competition for the reactive surface.

To explore further the PCET process, we performed DFT calculations with Quantum Espresso (QE), a package for computing the properties of periodic systems. ${ }^{18}$ Association energy $\left(E_{\text {ads }}\right)$ between substrates and solid surfaces is a computational routine in the $\mathrm{QE}$ package. Thus, we computed 205 the energy released by the interaction between THF and $\mathrm{TiO}_{2}(101)$ anatase surface, under both conditions neutral or positively charged $\mathrm{TiO}_{2}$ surface. As metals show limited influence on the reactivity of the hole (Fig. 1), only the $\mathrm{TiO}_{2}$ surface was included in this approximation. Fig. 3 shows top and side view for a THF adsorbed on neutral $\mathrm{TiO}_{2}$. A similar configuration is computed for positively charged $\mathrm{TiO}_{2}$. Importantly, when the surface is positive the stabilization increases by $23.8 \mathrm{kcal} / \mathrm{mol}$. As the positive surface is a reasonable model for the $\mathrm{TiO}_{2}$-hole, this indicates the beginning of 215 the electron transfer process in which THF neutralizes the highly electrophilic hole. In polar media, this becomes the nascent PCET process in which the electron is donated to the hole and the proton stabilized by the solvent, a type of PCET described as dystonic to emphasize the different destination 220 of proton and electron. As expected by their molecular geometry, di-ethers do not show any preferred adsorption configuration compared to those with one oxygen (Fig. S13). Further thermodynamic details can be found in table S4.

Free radical formation for other non-activated $\mathrm{C}-\mathrm{H}$ sys225 tems was also shown with pure toluene or acetonitrile. For toluene Fig. S14 shows the formation of bibenzyl and ring coupling products from benzyl radicals. TEMPO also traps $\cdot \mathrm{CH}_{2} \mathrm{CN}$ from acetonitrile (Fig. S15). Although the $\mathrm{C}-\mathrm{H}$ bond dissociation energy (BDE) in acetonitrile (92 $\left.230 \mathrm{kcal} / \mathrm{mol}^{19}\right)$ is within the same range of BDE in toluene or $\alpha$ - 
$\mathrm{C}-\mathrm{H}$ in ethers, this result is appealing as the electron poor nature of $\mathrm{CH}_{3} \mathrm{CN}$ makes it rather inert to typical electrophilic radicals, ${ }^{20}$ such as ${ }^{\mathrm{t}} \mathrm{BuO}$.



Figure 3: Computed adsorption configuration of THF on anatase $\mathrm{TiO}_{2}(101)$ surface. Side (left) and top view.

In conclusion, hole trapping in $\mathrm{TiO}_{2}$, and probably in other semiconductors of comparable band gap, serves as an excellent free radical source, easy to control, use, and separate once the radical source is no longer required. While pristine $\mathrm{TiO}_{2}$ in its anatase form can perform this function, decorating the material enhances free radical generation by a factor of $c a$. 5 , and is attributed to electron trapping by the metallic center that reduces the rate of electron-hole recombination. While we focused on ethers, the rich SED literature suggests that many molecules can perform similarly, as demonstrated here with toluene and acetonitrile. Numerous molecules should be capable of donating an electron to the VB hole, thus being excellent candidates for a remarkable clean and efficient source of free radicals.

\section{ASSOCIATED CONTENT}

Supporting Information. SI available free of charge on the ACS Publications website. Experimental and computational methodologies, additional kinetic information, EPR trapping data, MS spectra of the products.

\section{Corresponding Authors}

*E-mail: jscaiano@uottawa.ca (J.C.S.)

*E-mail: anabel.lanterna@icloud.com (A.E.L.)

\section{ACKNOWLEDGMENT}

wis work was supported by the Natural Sciences and Engineering Research Council of Canada, the Canada Foundation for Innovation, the Canada Research Chairs Program, Canada's International Development Research Centre (IDRC) and the German National Academy of Sciences Leopoldina (Grant No. LPDS 2017-15 to P.C.).

\section{REFERENCES}

1. Yi, H.; Zhang, G. T.; Wang, H. M.; Huang, Z. Y.; Wang, J.; Singh, A. K.; Lei, A. W., Recent Advances in Radical C-H Activation/Radical CrossCoupling. Chem. Rev. 2017, 117 (13), 9016-9085.

270 2. Zhang, S. Y.; Zhang, F. M.; Tu, Y. Q., Direct $\mathrm{Sp}(3)$ alpha-C-H activation and functionalization of alcohol and ether. Chem. Soc. Rev. 2011, 40 (4), 1937-1949.

3. Taylor, R. D.; MacCoss, M.; Lawson, A. D. G., Rings in Drugs. J. Med. Chem. 2014, 57 (14), 5845-5859.

275 4. Martins, P.; Jesus, J.; Santos, S.; Raposo, L. R.; Roma-Rodrigues, C.; Baptista, P. V.; Fernandes, A. R., Heterocyclic Anticancer Compounds: Recent Advances and the Paradigm Shift towards the Use of Nanomedicine's Tool Box. Molecules 2015, 20 (9), 16852-16891.
5. Jin, J.; MacMillan, D. W. C., Direct alpha-Arylation of Ethers through 280 the Combination of Photoredox-Mediated C-H Functionalization and the Minisci Reaction. Angew. Chem. Int. Edit. 2015, 54 (5), 1565-1569. Shaw, M. H.; Twilton, J.; MacMillan, D. W. C., Photoredox Catalysis in Organic Chemistry. J. Org. Chem. 2016, 81 (16), 6898-6926.

6. Mayer, J. M., Understanding Hydrogen Atom Transfer: From Bond

285 Strengths to Marcus Theory. Acc. Chem. Res. 2011, 44 (1), 36-46.

7. Gaya, U. I.; Abdullah, A. H., Heterogeneous photocatalytic degradation of organic contaminants over titanium dioxide: A review of fundamentals, progress and problems. J. Photochem. Photobio., C: Photochem. Rev. 2008, 9 (1), 1-12.

290 8. Morsella, M.; d'Alessandro, N.; Lanterna, A. E.; Scaiano, J. C., Improving the Sunscreen Properties of $\mathrm{TiO}_{2}$ through an Understanding of Its Catalytic Properties. ACS Omega 2016, 1 (3), 464-469. Sendra, M.; Sanchez-Quiles, D.; Blasco, J.; Moreno-Garrido, I.; Lubian, L. M.; PerezGarcia, S.; Tovar-Sanchez, A., Effects of $\mathrm{TiO}_{2}$ nanoparticles and sunscreens 295 on coastal marine microalgae: Ultraviolet radiation is key variable for toxicity assessment. Environ. Int. 2017, 98, 62-68. Hanigan, D.; Truong, L.; Schoepf, J.; Nosaka, T.; Mulchandani, A.; Tanguay, R. L.; Westerhoff, P., Trade-offs in ecosystem impacts from nanomaterial versus organic chemical ultraviolet filters in sunscreens. Water Res. 2018, 139, 281-290.

300 9. Tyagi, A.; Yamamoto, A.; Kato, T.; Yoshida, H., Bifunctional property of Pt nanoparticles deposited on $\mathrm{TiO}_{2}$ for the photocatalytic $\mathrm{sp}(3) \mathrm{C}$ $\mathrm{sp}(3) \mathrm{C}$ cross-coupling reactions between THF and alkanes. Catal. Sci. Technol. 2017, 7 (12), 2616-2623. Marina, N.; Lanterna, A. E.; Scaiano, J. C., Expanding the Color Space in the Two-Color Heterogeneous 305 Photocatalysis of Ullmann C-C Coupling Reactions. ACS Catal. 2018, 8 (8), 7593-7597.

10. Fuchigami, T.; Fuchigami, T. a., Fundamentals and Applications of Organic Electrochemistry : Synthesis, Materials, Devices. Wiley: Hoboken, 2014.

310 11. Schneider, J.; Bahnemann, D. W., Undesired Role of Sacrificial Reagents in Photocatalysis. Journal of Physical Chemistry Letters 2013, 4 (20), 3479-3483.

12. Wang, B.; Durantini, J.; Nie, J.; Lanterna, A. E.; Scaiano, J. C., Heterogeneous Photocatalytic Click Chemistry. J. Am. Chem. Soc. 2016, 315138 (40), 13127-13130.

13. Hernandez Mejia, C.; van Deelen, T. W.; de Jong, K. P., Activity enhancement of cobalt catalysts by tuning metal-support interactions. Nat Commun 2018, 9 (1), 4459.

14. Malatesta, V.; Scaiano, J. C., Absolute Rate Constants for the Reactions

320 of Tert-Butoxyl with Ethers - Importance of the Stereoelectronic Effect. J. Org. Chem. 1982, 47 (8), 1455-1459.

15. Hainer, A. S.; Hodgins, J. S.; Sandre, V.; Vallieres, M.; Lanterna, A. E.; Scaiano, J. C., Photocatalytic Hydrogen Generation Using MetalDecorated $\mathrm{TiO}_{2}$ : Sacrificial Donors vs True Water Splitting. ACS Energy 325 Lett. 2018, 3 (3), 542-545.

16. Ciriano, M. V.; Korth, H. G.; van Scheppingen, W. B.; Mulder, P., Thermal stability of 2,2,6,6-tetramethylpiperidine-1-oxyl (TEMPO) and related N-alkoxyamines. J. Am. Chem. Soc. 1999, 121 (27), 6375-6381.

17. Orlov, Y. D.; Chernova, E. M.; Turovtsev, V. V., Enthalpies of 330 formation of organic free radicals of alcohol and ether derivatives. Russ. Chem. Bull. 2010, 59 (10), 2009-2013.

18. Giannozzi, P.; Baroni, S.; Bonini, N.; Calandra, M.; Car, R.; Cavazzoni, C.; Ceresoli, D.; Chiarotti, G. L.; Cococcioni, M.; Dabo, I.; Dal Corso, A.; de Gironcoli, S.; Fabris, S.; Fratesi, G.; Gebauer, R; Gerstmann, U.; 335 Gougoussis, C.; Kokalj, A.; Lazzeri, M.; Martin-Samos, L.; Marzari, N.; Mauri, F.; Mazzarello, R.; Paolini, S.; Pasquarello, A.; Paulatto, L.; Sbraccia, C.; Scandolo, S.; Sclauzero, G.; Seitsonen, A. P.; Smogunov, A.; Umari, P.; Wentzcovitch, R. M., QUANTUM ESPRESSO: a modular and opensource software project for quantum simulations of materials. J. Phys.:

340 Condens. Matter 2009, 21 (39).

19. Holmes, J. L.; Lossing, F. P.; Mayer, P. M., The Effects of Methyl Substitution on the Structure and Thermochemistry of the Cyanomethyl Radical and Cation. Chem. Phys. Lett. 1993, 212 (1-2), 134-140.

20. De Vleeschouwer, F.; Van Speybroeck, V.; Waroquier, M.; Geerlings,

345 P.; De Proft, F., Electrophilicity and nucleophilicity index for radicals. Org. Lett. 2007, 9 (14), 2721-2724. 
Toc Graphic

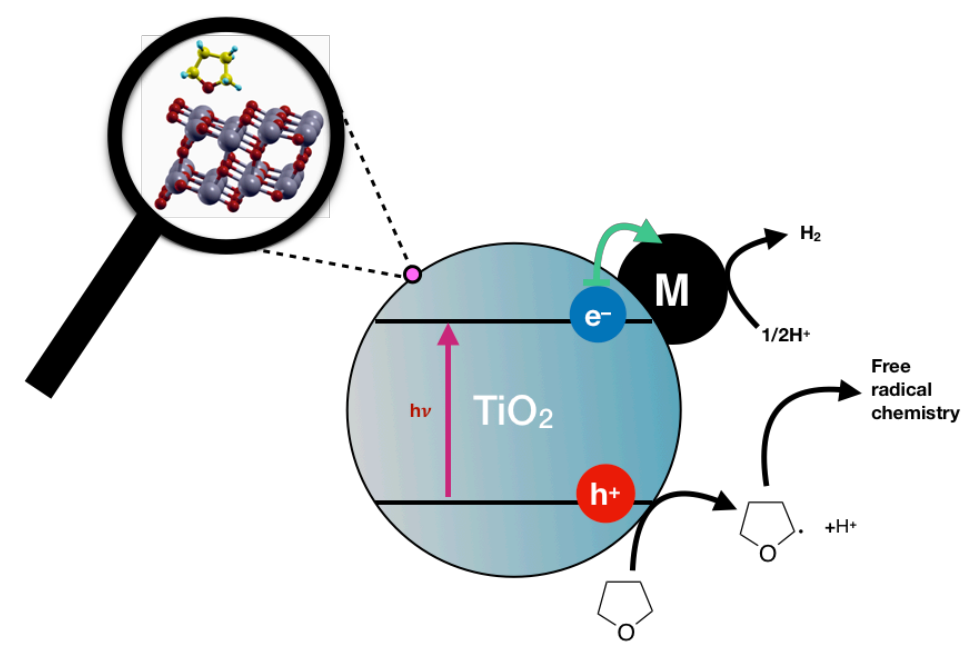

